\title{
HYPERTENSION
}

\section{Nogo-B regulates BP homeostasis}

Nogo-B regulates sphingolipid biosynthesis within the endothelium to modulate blood pressure (BP) and could open up new avenues for pharmacologic intervention in hypertension, according to new findings by Annarita Di Lorenzo and colleagues. "The importance of sphingolipid signalling in cardiovascular diseases, including hypertension, has been recognized," says Di Lorenzo, "but the molecular connection between the sphingolipid pathway and Nogo-B was previously unknown."

Nogo-B is one of three RTN4 isoforms, and is highly expressed in endothelial cells, whereas Nogo-A and Nogo-C are abundantly expressed in the central nervous system. In their study, the researchers found that Nogo-B might have a specific role in $\mathrm{BP}$ homeostasis after observing that mice deficient in Nogo-A and Nogo-B isoforms (Nogo-A/B), both systemically and specifically in endothelial cells, were hypotensive and had elevated sphingolipid levels in endothelial cells compared to controls.
The contractility of resistance vessels was maintained.

The Nogo-A/B mice were resistant to the effects of chronic infusion of angiotensin II (Ang-II) to induce hypertension. However, their BP did rise to that of Ang-II-treated controls after administering an inhibitor of serine palmitoyltransferase-the ratelimiting enzyme of de novo sphingolipid synthesis. Nogo-B was also shown to bind and inhibit the activity of serine palmitoyltransferase in vitro and in vivo, thus implicating it as a direct regulator of sphingolipid production. The researchers hope to further their understanding of how Nogo-B regulates sphingolipid signalling, and identify ways by which this pathway can be therapeutically manipulated.

Jessica K. Edwards

Original article Cantalupo, A. et al. Nogo-B regulates endothelial sphingolipid homeostasis to control vascular function and blood pressure. Nat. Med. doi:10.1038/ $\underline{\mathrm{nm} .3934}$ 